\title{
Irreversible Lattice Defects Formed by Martensitic Transformation Cycles in a Nearly Thermoelastic Fe-Pt Alloy*
}

\author{
By M. Umemoto** and C. M. Wayman***
}

\begin{abstract}
Irreversible lattice defects left behind in the parent phase after a complete transformation cycle (parent-martensite-parent) in nearly thermoelastic specimens were studied by transmission electron microscopy. A commonly observed configuration is that of parallel dislocations lying on the (101) $f$ plane and along the $[\overline{101}]_{f}$ direction, forming pairs with other dislocations which lie on adjacent $(101)_{f}$ planes some 5 to $10 \mathrm{~nm}$ apart. Another structure observed is what is supposed to be the junction plane of pairs of martensite variants once exising at a low temperature. The junction plane of a pair of variants which formed a wedge morphology contains two directions of line defects (probably dislocations), each of which corresponds to the intersections of the transformation twin planes of each vaiant and the junction plane. The junction plane of a pair of variants which formed a spear type morphology shows a single direction of line defects, and the junction region of a pair of variants which formed a kink type morphology contains a cluster of dislocations.
\end{abstract}

(Received December 28, 1977)

\section{Introduction}

A thermoelastic martensitic transformation is characterized by a small thermal hysteresis, a mobile interface and thermoelastic equilibrium at the interface. Thus, the ideal thermoelastic martensitic transformation is considered not to cause the generation of irreversible lattice defects in both the foward and reverse transformations. On the other hand, the immobile interface and large thermal hysteresis in non-thermoelastic transformations appears to be related with the formation of irreversible defects. The understanding of the structure and origin of these irreversible lattice defects is important to obtain a better understanding of not only thermoelastic and non-

* This paper is based upon a thesis submitted by M. Umemoto in partial fulfilment of the requirement of the degree of Doctor of Philosophy at the University of Illinois at Urbana-Champaign.

** Formerly Research Assistant at the University of Illinois at Urbana-Champaign, Urbana, Illinois, is now with the Department of Metal Science and Technology, Kyoto University, Kyoto 606, Japan.

*** Metallurgy at the University of Illinois at Urbana-Champaign. thermoelastic transformations but also the mode of reverse transformation and microstructual reproducibility ${ }^{(1) \sim(3)}$.

Several investigations have been concerened with the irreversible defects left behind in the parent phase after a complete or incomplete transformation cycle. Krauss ${ }^{(4)}$ investigated the substructure introduced in austenite by the reverse martensitic transformation in a nonthermoelastic $\mathrm{Fe}-\mathrm{Ni}$ alloy. It was reported that the reversed austenite is quite imperfect, containing a high concentration of tangled and jogged dislocations with interspersed loops. The dislocation density was about a factor of ten greater than that observed in the retained austenite in partially transformed specimens. Kajiwara and Owen ${ }^{(2)}$ studied semi-thermoelastic $\mathrm{Fe}-27$ at $\% \mathrm{Pt}$ and $\mathrm{Fe}-25$ at $\% \mathrm{Pt}$ alloys and observed that the $\alpha-\gamma$ transformation produces austenite regions with a high density of dislocations. These regions are separated from the surrounding untransformed austenite by arrays of dislocation loops lying near the interface between the untransformed austenite and the original martensite plate. Such dislocations have a Burgers vector which is the same as that of the usual slip dislocation in austenite. They also studied a near thermoelastic Fe25 at $\% \mathrm{Pt}$ alloy and observed that only a few

1978 Vol. 19

Trans. JIM 
dislocations are associated with the martensite habit plane trace in the plane of the specimen foil. Recently, Maki and Wayman ${ }^{(5)}$ showed arrays of dislocation loops around "thin plate" martensite in $\mathrm{Fe}-\mathrm{Ni}-\mathrm{C}$ alloys. According to their observations, the dislocations around the thin plate martensite consisted of looped dislocations, the long portions of which being parallel to each other. The spacing of the dislocation loops corresponds to the martensite twin width and the long portions of the loops lie on the particular $(101)_{f}$ plane with Burgers vector $[\overline{101}]_{f}$, these corresponding to the $(112)_{b}$ twin plane and the $[111]_{b}$ twin direction in the martensite by the Bain correspondencet.

The above observations have clarified some of the details of the irreversible defects but, at the same time, have raised other questions. A difficulty in carrying out impeding further studies is that the observed structure is too complicated with a substantial number of defects. Since an ideal thermoelastic alloy does not produce irreversible defects, it is considered that the more a specimen possesses the thermoelastic nature, the fewer irreversible defects are produced during a transformation cycle. If so, the lower density of irreversible defects in nearly thermoelastic specimens is expected to give a more simplified picture of the nature and origin of the observed irreversible defects.

The purpose of the present work is thus to examine in detail the irreversible lattice defects remaining in the parent phase after a complete transformation cycle in nearly thermoelastic $\mathrm{Fe}-\mathrm{Pt}$ specimens, using transmission electron microscopy.

\section{Experimental Procedure}

An alloy of iron and platinum containing nominally 24 at $\% \mathrm{Pt}$ was prepared from $99.992 \%$ iron rods and $99.99 \%$ platinum wire. The specimens were induction melted and remelted under argon in an alumina crucible at about $1650^{\circ} \mathrm{C}$ and kept in the molten stage at least $30 \mathrm{~min}$ for homogenization. The assolidified ingots were cold worked (about

$\dagger$ Subscripts $f$ and $b$ apply to the austenite $(f c c)$ and martensite $(b c c)$ respectively.
$5 \%)$ and homogenized $\left(1200^{\circ} \mathrm{C}\right.$ for $\left.7 \mathrm{~d}\right)$ in quartz capsules evacuated to $1.3 \times 10^{-3} \mathrm{~Pa}$ and then back filled to $2 \times 10^{4} \mathrm{~Pa}$ with pure argon.

Specimens in the form of $3 \mathrm{~mm}$ diameter discs were cut from sheets rolled to $0.5 \mathrm{~mm}$, after which the individual discs were sealed off in quartz capsules evacuated to $1.3 \times 10^{-3}$ $\mathrm{Pa}$, austenitized at $1200^{\circ} \mathrm{C}$ for $5 \mathrm{~h}$ and subjected to an ordering treatment at $550^{\circ} \mathrm{C}$ for $150 \mathrm{~h}$. The ordered samples were then quenched into liquid nitrogen to transform them into martensite. Upon heating back to room temperature all the martensite reversed to austenite. The specimen were then thinned to $0.25 \mathrm{~mm}$ on silicon carbide paper followed by jet polishing using a solution of $60 \% \mathrm{H}_{3} \mathrm{PO}_{4}$ and $40 \%$ $\mathrm{H}_{2} \mathrm{SO}_{4}$ kept at $10^{\circ} \mathrm{C}$. Good polishing was obtained only this solution, although many solutions known for electropolishing stainless steels were tried. The voltage across the specimen was kept at $40 \mathrm{~V}$ and the current at that condition was $200 \mathrm{~mA}$. The polishing time proved to be extraodinaly long, being typically 15-30 h. Specimens were observed at room temperature in a Hitachi HU-12 electron microscope operating at $100 \mathrm{kV}$.

\section{Experimental Results and Discussion}

\section{General features of irreversible defects}

Figure 1 shows a typical resistivity-temperature curve for the specimens used in this experiment. The $M_{s}$ and $A_{f}$ temperatures were measured as -94 and $-48^{\circ} \mathrm{C}$, respectively, and the amount of thermal hysteresis was about $30^{\circ} \mathrm{C}$.

To avoid any confusion between the defects caused by the transformation cycle and those caused by other factors (during polishing, etc.), austenitic specimens which were polished just after the ordering treatment and without transformation were initially examined. It was confirmed that such austenitic specimens contain few defects after the heat treatment and jet polishing. This indicates that the defects observed in the specimen after a transformation cycle are actually caused by the transformation cycle. 


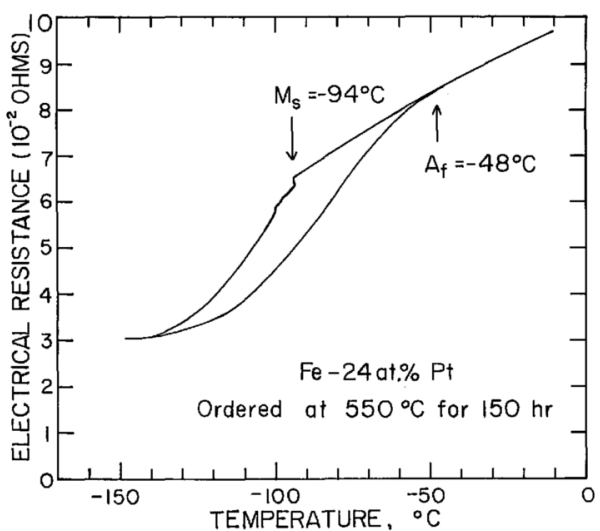

Fig. 1 Electrical resistivity vs temperature curve showing a complete transformation hysteresis loop for the 1st cycle in an $\mathrm{Fe}-24$ at \% Pt alloy ordered at $550^{\circ} \mathrm{C}$ for $150 \mathrm{~h}$.

Photograph 1(a) and (b) show representative transmission electron micrographs of the defects observed in a specimen which experienced a complete thermal cycle before polishing. It is to be noted that the distribution of the dislocations in the observed area is not uniform. Some regions are completely dislocation-free and others show relatively uniformly distributed dislocations lying along a common direction. Several straight bands thought to be a junction plane between martensite plates (when the specimen was in the martensite condition) are also observed. Photograph 1(b) shows a region with a high density of dislocations. The configuration of these regions of clustered dislocations is different from place to place and no typical distributions are observed. The specimens used in the present investigation are nearly thermoelastic and the martensite is considered to form cooperatively to minimize the transformation shape strain. However, the observed dislocation clusters indicate the existence of localized highly deformed regions.

Photograph 1(c) shows, as occasionally observed, a retained martensite plate. This was confirmed by electron diffraction and a dark field image. The amount of martensite in the observed area was well less than $1 \%$. The $\mathrm{A}_{\mathrm{f}}$ temperature for this alloy as measured by electrical resistivity-temperature measurements is $-48^{\circ} \mathrm{C}$ and it is considered that such a small amount of martensite as observed under the electron microscope at room temperature was not detected by the resistivity measurement.

As is noted, there are many configurations of irreversible defects. Some of them are complicated and far from being fully understood while several simple situations are possible to understand from crystallographic knowledge of the martensite transformation using electron diffraction and trace analysis.

\section{Dislocation structures}

Photograph 2 shows one of the commonly observed dislocation arrays. All the dislocations
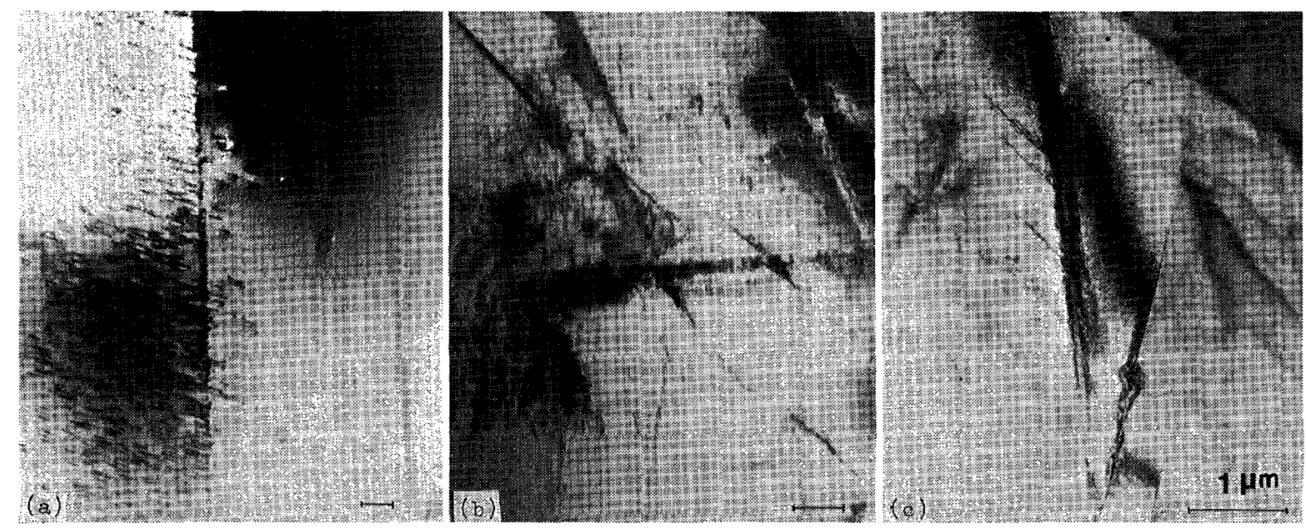

Photo. 1 Transmission electron micrographs showing the general features of irreversible lattice defects observed in an Fe-24 at \% Pt alloy ordered at $550^{\circ} \mathrm{C}$ for $150 \mathrm{~h}$, followed by a complete transformation cycle. (a) relatively uniformly distributed dislocations; (b) high density of dislocations; (c) retained martensite. 


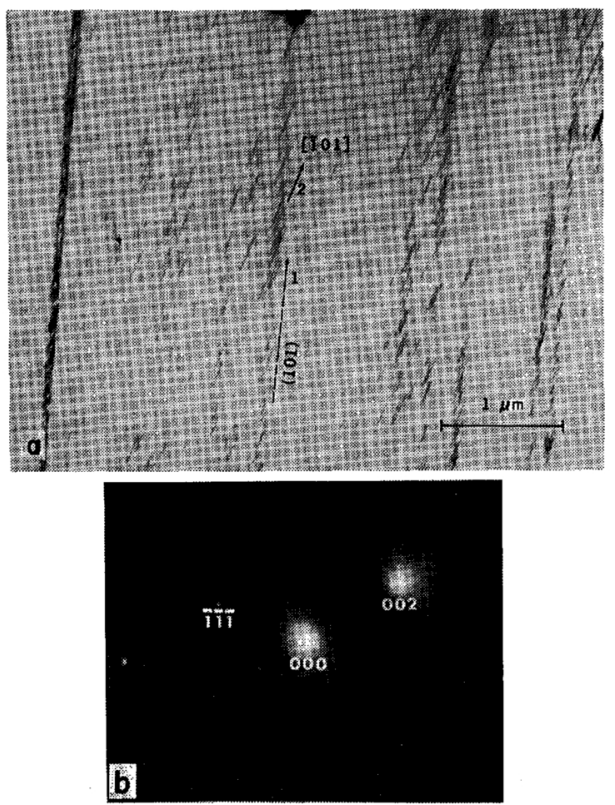

Photo. 2 Transmission electron micrograph showing dislocation arrays on $(101)_{f}$ plane forming pairs with dislocations lying on a parallel $(101)_{f}$ plane.

(a) bright field image; (b) diffraction pattern from (a) with zone axis $[\mathbf{1 1 0}]_{f}$.

in the observed region are nearly straight, parallel to each other and lying on a common plane. The corresponding electron diffraction pattern shows that the foil is in an approximate $[\overline{1} 10]_{f}$ orientation. From trace analysis, trace 1 is parallel to both $(101)_{f}$ and $(011)_{f}$. These two planes are in a symmetrical mirror position with respect to the specimen surface. The direction of the dislocation is obtained as $[\overline{101}]_{f}$ when the plane is chosen as $(101)_{f}$ or $[0 \overline{1} 1]_{f}$ for $(011)_{f}$. It was confirmed that all the dislocations lie on one of these planes (not both) by tilting the specimen. It should be noted that $\{110\}_{f}$ is not a regular slip plane in the austenite. It is of interest that the $(101)_{f}$ plane is related to the martensite as the $(112)_{b}$ twin plane and $[\overline{101}]_{f}$ corresponds to the $[\overline{1} 11]_{b}$ twin direction from the Bain correspondence (as does the $(011)_{f}[0 \overline{1} 1]_{f}$ ).

The dislocations are not evenly spaced on the (101) plane and also the (101) $)_{f}$ planes containing the dislocations are not uniformly distributed. It is to be noted that all the dislocations in the micrograph appear as paired with each dislocation in the pair lying on different $(101)_{f}$ planes spaced about 5 to 10 $\mathrm{nm}$ apart. Furthermore, all the dislocations on a given $(101)_{f}$ plane form a pair with dislocations lying on a parallel $(101)_{f}$ plane which contains the same number of dislocations as the former. "U" shaped and completely looped dislocations are also seen in the figure. The vertical portions of the " $U$ " shaped dislocations are parallel to $[\overline{101}]_{f}$ and are considered to lie on the $(101)_{f}$ plane.

Photograph 3 shows that the dislocations in a pair are of opposite sign. The difference in the sign of the Burgers vector of the dislocation can be recognized by complementary behaviour of the fringes along the dislocation (predicted by the dynamic theory). As is seen in the Photo. 3, the sense of the black-white oscillations at the ends of the dislocations in a pair is opposite, indicating that the dislocations are of opposite $\operatorname{sign}^{(6)}$. It is further noted that all the dislocations on a given $(101)_{f}$ plane have the same sign and those on the adjacent $(101)_{f}$ plane have the opposite sign.

A schematic diagram of the observed dislocation structures is shown in Fig. 2, where parallel dislocations lying on the $(101)_{f}$ planes form a pair with those which lie on the adjacent $(101)_{f}$ plane. The sign of the dislocations on each $(101)_{f}$ plane is the same and the sign of those on the adjacent $(101)_{f}$ plane is opposite. "U" shaped, negative "U" shaped and completely looped dislocations are also shown.

The above dislocations are considered to be portions of large elongated loops associated with the martensite interface, according to the

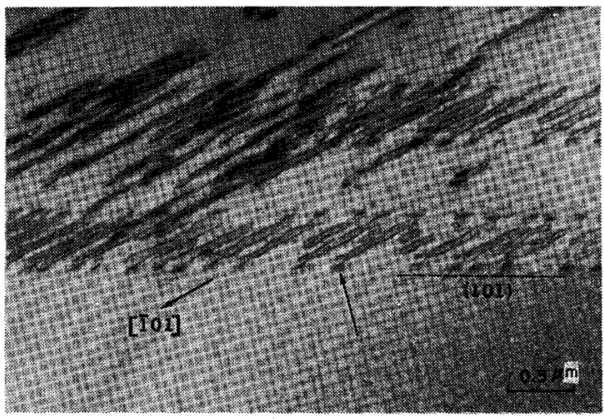

Photo. 3 Transmission electron micrograph showing that the paired dislocations as in Photo. 2 are of opposite sign. 


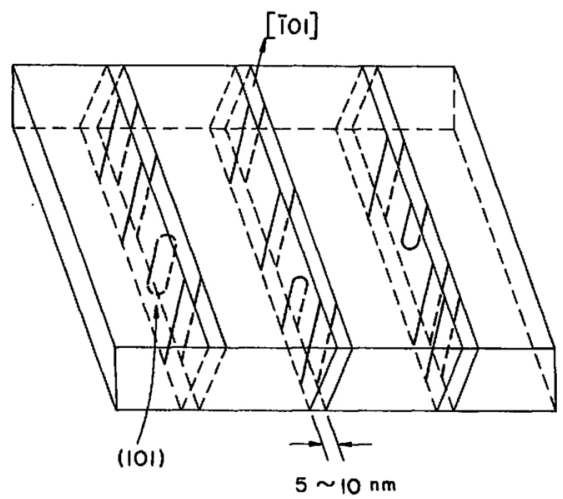

Fig. 2 Schematic drawing of observed dislocation configurations. Indices refer to austenite phase.

following argument. As observed for the dislocations associated with the martensite interface in $\mathrm{Fe}-\mathrm{Ni}-\mathrm{C}^{(5)}$ alloys the dislocations observed in the present study lie on the $(101)_{f}$ plane which correspond to the (112) $b$ twin plane in the martensite. The opposite sign of the paired dislocations and the complete and incomplete dislocations loops suggest that all the paired dislocation in Photos. 2 and 3 are portions of large elongated loops. The distance between the two $(101)_{f}$ planes which contain dislocations of opposite sign is 5 to $10 \mathrm{~nm}$, which is of the order of the transformation twin thickness in the thin plate martensite in ferrous alloys ${ }^{(7)(8)}$. Although the Burgers vector of the dislocations was not determined uniquely (either $[\overline{101}]_{f}$ or $[0 \overline{1} 1]_{f}$ ) in the present study, from the above similarity the dislocations in Photos. 2 and 3 are considered to be portions of large elongated loops associated with the martensite interface.

Kajiwara and Owen $^{(2)}$ observed similar arrays of dislocation loops in semi-thermoelastic Fe-Pt alloys. They reported that these loops are lying in the original interface and proposed a model of the origin of these dislocations ${ }^{(9)}$. In the present investigation the original interfaces were not observed since the specimens were completely transformed into martensite. Thus, it is difficult to compare the mentioned dislocation loops with those observed by them. Kajiwara and Owen also observed dislocations within the plate of austenite which was originally a martensite plate. These are the dislocations which may correspond to what was ob- served in the present investigation. However, since they did not identify the planes on which these dislocations lie, it is difficult to discuss the similarlity between their dislocations and those in the present study.

\section{Pairs of variants}

It was recently shown ${ }^{(10)}$ that there are three types of pairs formed by the four martensite plate variants in a plate group: the wedge (A-B), spear (A-C) and kink (A-D) type pairs (see Fig. 3). The wedge forms a self-accommodating pair. The junction plane of this pair bisects with an acute angle the habit planes of the variants and its normal is situated on the $(0 \overline{1} 1)_{f}$ zone a few degrees away from the $(011)_{f}$ pole (in the case of the plate group about $(011)_{f}$ ). This junction plane is not a twin plane of the variants. The spear type pair also forms a self-accommodating pair. The junction plane of this pair is (011) $)_{f}$ and this bisects also with an acute angle the habit planes of the variants. This junction plane is a twin plane of the variants. The kink type pair is not self-accom-
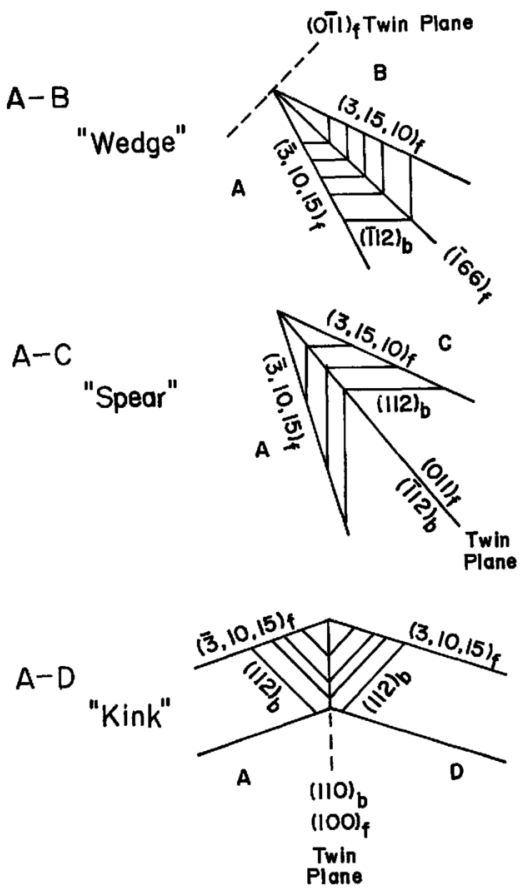

(c)

Fig. 3 Schematic drawings corresponding to A-B, A-C and A-D type martensite pairs as discussed in text. 
modating. The junction plane of this pair is a nominal $(100)_{f}$ plane and this bisects with an obtuse angle the habit planes of the variants. This is also a twin plane of the variants.

Photograph 4 shows a region which is considered to form a spear type pair (A-C) when the specimen was in the martensite condition. From trace analysis, Fig. 4, trace 1 is parallel to the intersection of $(101)_{f}$ and the foil plane, and the dislocations on this plane lie along $[\overline{101}]_{f}$. These dislocations are the type observed in Photos. 2 and 3 and are considered to be dislocations associated with the martensite interface. Trace 3 is parallel to the intersection of the foil plane and $(011)_{f}$ which corresponds to the junction plane of a spear type pair. By tilting the specimen, the upper right region reveals similar dislocations on the $(\overline{110})_{f}$ plane (Photo. 5). From the Bain correspondence these planes which contain the above dislocations, $(101)_{f}$ and $(\overline{1} 10)_{f}$, become a $(112)_{b}$ transformation twin plane in the $\mathrm{C}$ and $\mathrm{A}$ variants. What
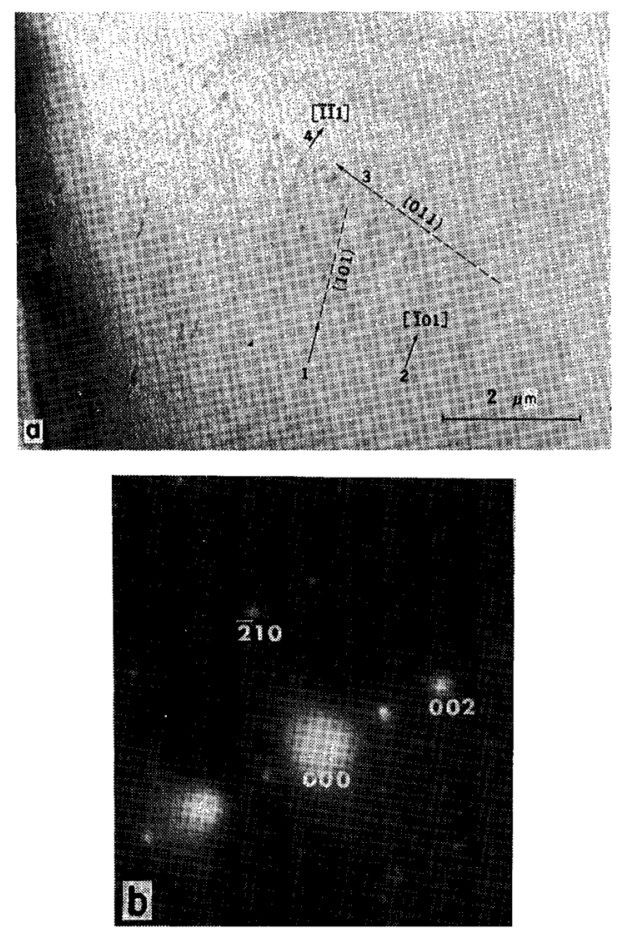

Photo. 4 Transmission electron micrograph showing a region which appeared to form an A-C type pair when the specimen was in the martensite condition. (a) Bright field image; (b) diffraction pattern from (a) with zone axis $[120]_{f}$.

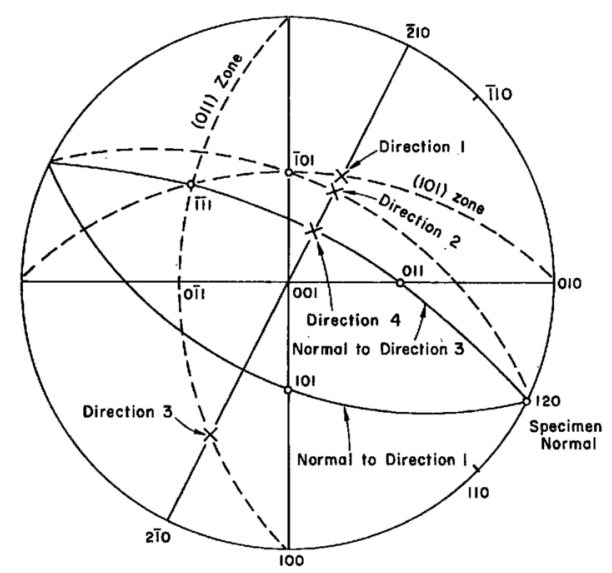

Fig. 4 Stereographic analysis of Photo. 4. Indices refer to austenite phase.

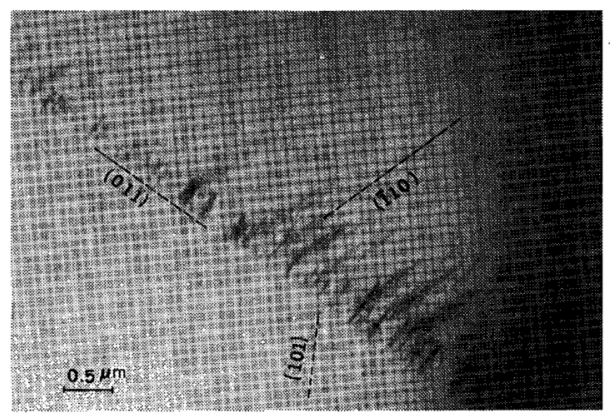

Photo. 5 Transimission electron micrograph showing dislocation arrays in the upper right region of Photo. 4 as revealed after tilting the specimen.

appears to be dislocations on the $(011)_{f}$ plane lie along $[\overline{1} 11]_{f}$ which is parallel to the intersection of the junction plane and the transformation twin planes. Many of the dislocations are in contact at the junction plane.

Photograph 6 shows that another example of the region which corresponds to a spear type pair when the specimen was in the martensite condition. From the diffraction pattern and trace analysis, traces 1,2 and 3 are parallel to $(\overline{1} 10)_{f},(011)_{f}$ and $(101)_{f}$, respectively. Since the plane of the foil is near $(\overline{111})_{f}$ these three planes are nearly perpendicular to the plane of the foil. The twin relation of the martensite variants with respects to the junction plane at low temperature is suggested by this micrograph.

Photograph 7 shows a region which was thought to correspond to a wedge (A-B) pair 


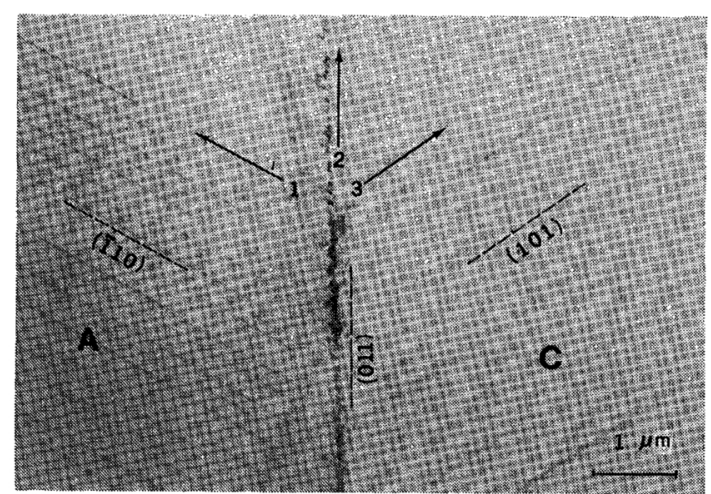

Photo. 6 Transmission electron micrograph showing another example of a region which corresponds to an A-C type pair when the specimen was in the martensite condition.
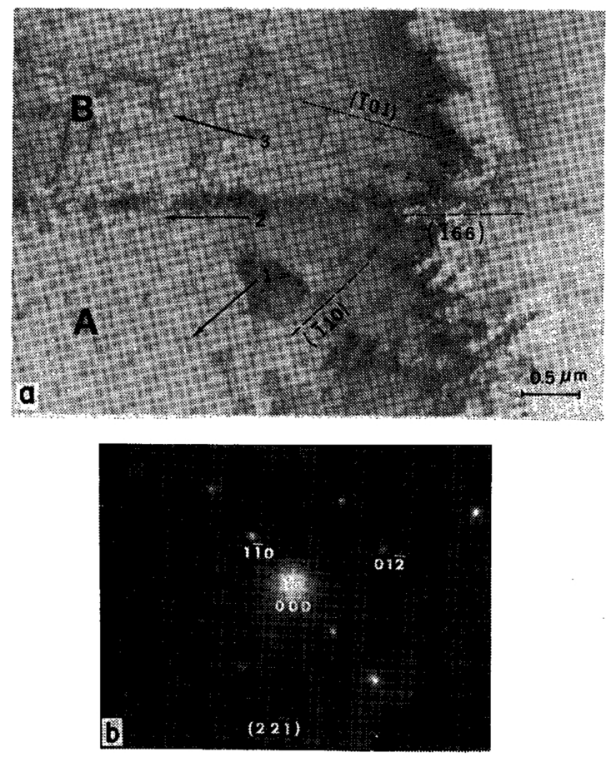

Photo. 7 Transmission electron micrograph showing a region believed to correspond to an A-B type pair junction when the specimen was in the martensite condition. (a) bright field image; (b) diffraction pattern from (a) with zone axis $[221]_{f}$.

when the specimen was in the martensite condition. From the diffraction pattern and trace analysis, traces 1 and 3 are the surface traces of $(\overline{1} 10)_{f}$ and $(\overline{101})_{f}$, respectively. These planes correspond to the transformation twin plane in the A and $\mathbf{B}$ variants. Trace 2 is nearly parallel to the surface trace of $(166)_{f}$ which is the junction plane of the A-B type pair. The A-B and A-C type pairs can clearly be dis- tinguished from the location of these planes. In the A-B type pair these three plane normals are nearly $60^{\circ}$ apart. In the A-C type pair the plane normals corresponding to the transformation twin planes are $120^{\circ}$ apart, and the junction plane bisects these planes. The three plane normals lie on the $[\overline{11} 1]_{f}$ zone.

As shown above, in an austenite specimen which experienced a complete transformation cycle, the regions at which the A-B and A-C type pairs formed can be recognized from the dislocations on the corresponding transformation twin planes in the original martensite variants and its junction plane. Although regions corresponding to the A-D type pair were not observed in the present investigation probably because of the low density of this type pair, it is expected that such regions can be also recognized in the same manner as for the A-B and A-C type pairs.

\section{Structures of the junction planes}

A schematic diagram of the A-B and A-C junctions is shown in Fig. 5. The calculated
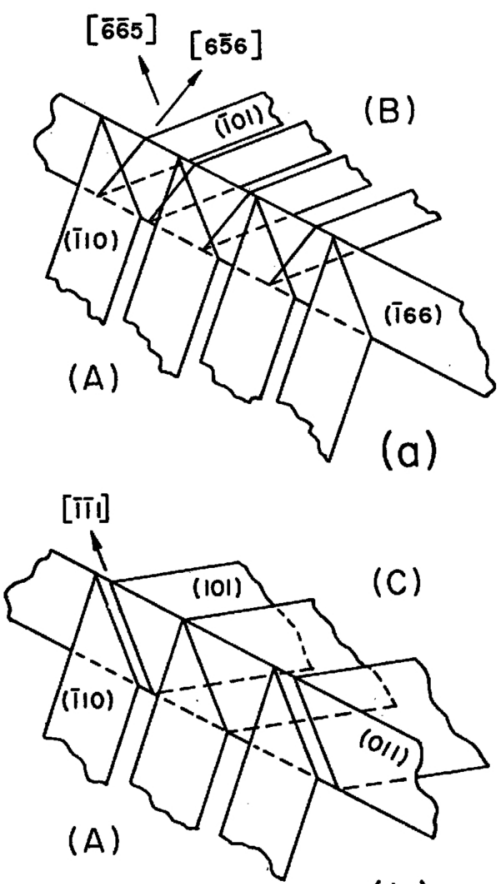

(b)

Fig. 5 Schematic diagram of A-B and A-C junctions. Indices refer to austenite phase. 
junction plane of the A-B type pair in $\mathrm{Fe}-\mathrm{Pt}$ alloys depends on the degree of order of the parent phase, and changes from $\{144\}_{f}$ in a disordered alloy to $\{166\}_{f}$ in a well ordered one. From the specimens studied in the present investigation the junction plane of an A-B pair is calculated to be close to $(166)_{f}$. From the Bain correspondence the transformation twin planes in the A and B variants are $(\overline{110})_{f}$ and $(\overline{101})_{f}$ in the parent crystal and these planes intersect the junction plane along $[\overline{6} 65]_{f}$ and $[656]_{f}$, respectively. The junction plane of the A-C pair is $(011)_{f}$ irrespective of the degree of order of the parent phase. The transformation twin planes of the A and $C$ variants are $(110)_{f}$ and $(101)_{f}$ and both planes intersect the junction along the $[\overline{1} \overline{1} 1]_{f}$. The junction plane of the A-D type pair is considered to be $\{100\}_{f}$. However, since this pair is not self-accommodating, the junction plane may not be such a simple plane. In fact, in the thermoelastic $\mathrm{Ni}-\mathrm{Al}^{(11)}$ and $\mathrm{Cu}-\mathrm{Zn}^{(12)}$ alloys the junction planes of this type pair have been observed as an irregular plane which consists of segments of $\{100\}_{p}$ planes.

Photograph 8 shows what is thought to be
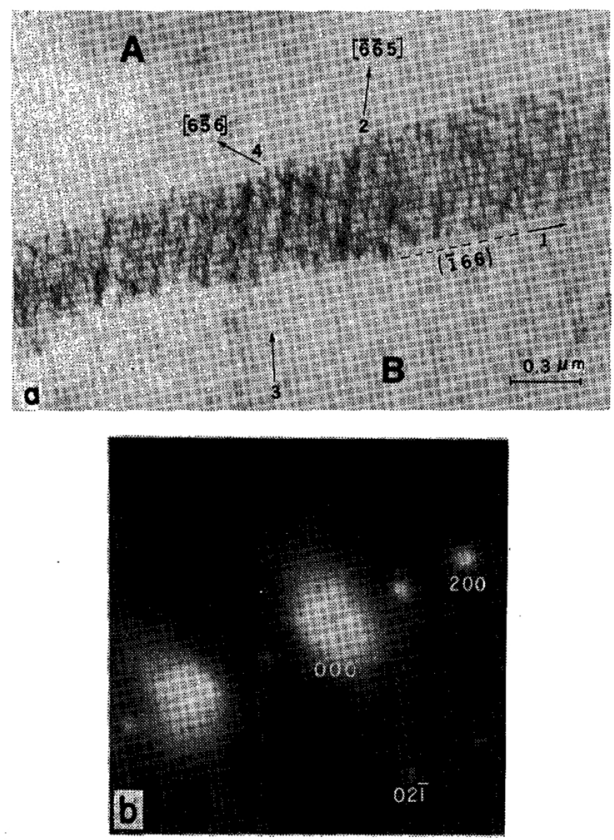

Photo. 8 Transmission electron micrograph showing what is supposed to be an A-B type junction plane.

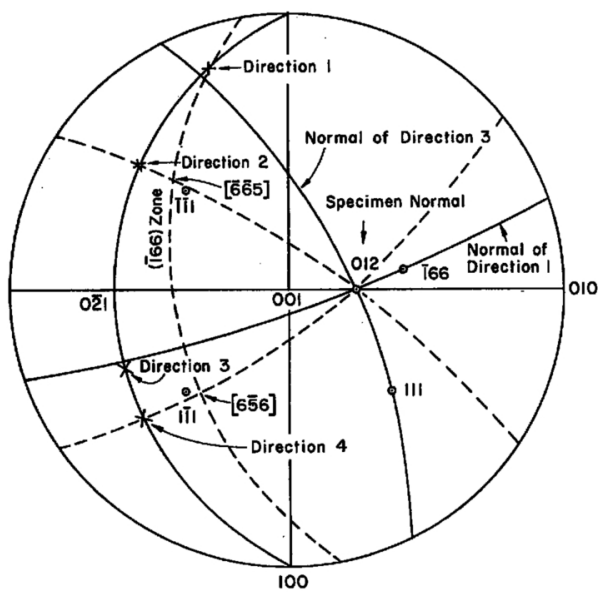

Fig. 6 Stereographic analysis of Photo. 8. Indices refer to austenite phase.

an A-B type junction plane observed in a completely cycled specimen. The electron diffraction pattern shows the foil is in an approximate $[012]_{f}$ orientation. From trace analysis, Fig. 6 , trace 1 is nearly parallel to $\left(\overline{1}^{166)_{f}}\right.$. The directions 2 and 4 in this plane are close to $[\overline{665}]_{f}$ and $[656]_{f}$, respectively. These traces are quite consistent with what is considered to be the junction of the $A$ and $B$ variants as schematically shown in Fig. 5 .

Photograph 9 shows a region where the A, B

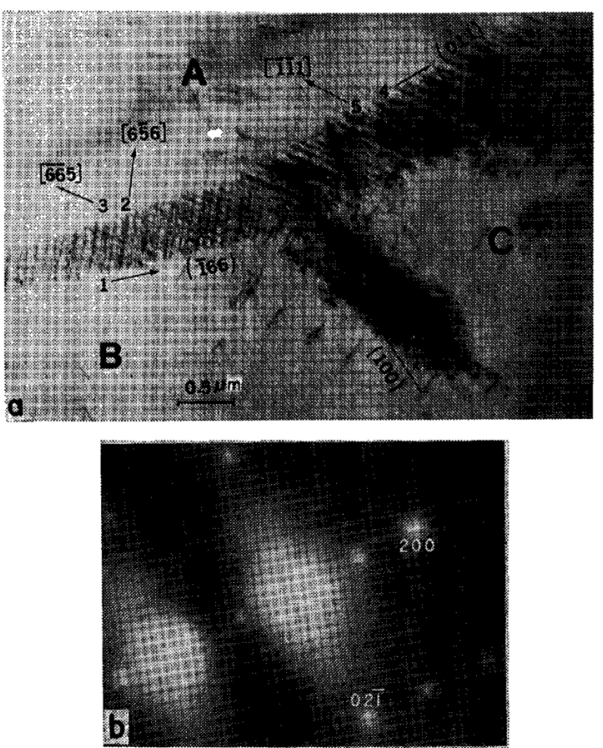

Photo. 9 Transmission electron micrograph showing a region where $A, B$ and $C$ variants of martensite is thought to be formed. 
and $\mathrm{C}$ variants of martensite were likely to be formed. From the electron diffraction pattern and trace analysis, trace 1 is close to being parallel to the junction plane of the $\mathrm{A}$ and $\mathrm{B}$ variants, $(\overline{1} 66)_{f}$, and directions 2 and 3 in this plane are along $[6 \overline{5} 6]_{f}$ and $[\overline{6} \overline{6} 5]_{f}$ which correspond to the intersections of the transformation twin planes of the $A$ and $B$ variants and the junction plane. Trace 4 is parallel to the junction plane of the A and $\mathrm{C}$ variants, $(011)_{f}$, and direction 5 in this plane is $[\overline{1} 11]_{f}$, which corresponds to the intersection of the transformation twin planes of the $\mathrm{A}$ and $\mathrm{C}$ variants with the junction plane. Near the boundary of the A-B and A-C junction planes, a dislocation cluster is observed essentially along the $(100)_{f}$ trace. This dislocation cluster is probably the region of junction of the $\mathrm{B}$ and $\mathrm{C}$ variants, indicating that the junction of $B$ and $C$ variants (A-D type) is not a simple plane, as expected.

The exact nature of the defects at the junction planes seen in Photos. 8 and 9 is not yet clear. However, from the observation that part of the dislocations on the corresponding transformation twin planes lie also in the junction plane (Photo. 4) it is quite probable that the observed junction plane consists of such trapped dislocations.

\section{Summary and Conclusions}

The irreversible lattice defects left behind in the parent phase after a complete transformation cycle (parent-martensite-parent) in nearly thermoelastic specimens were studied using transmission electron microscopy. In such specimens numerous defect structures are observed.

A commonly observed arrangement is that of parallel dislocations lying in the $(101)_{f}$ plane and along the $[\overline{1} 01]_{f}$ direction, forming pairs with other dislocations which lie on adjacent $(101)_{f}$ planes some 5 to $10 \mathrm{~nm}$ apart. From the Bain correspondence the (101) $f$ plane becomes the $(112)_{b}$ transformation twin plane, and $[\overline{101}]_{f}$ becomes the $[\overline{1} \overline{1} 1]_{f}$ transformation twin direction in the martensite. The sign of the dislocations on each $(101)_{f}$ plane is the same and the sign of those on the adjacent $(101)_{f}$ plane is opposite. " $U$ " shaped, negative " $U$ " shaped and completely looped dislocations are also observed among these dislocation arrays. From the similarrity of the above dislocation structures and that observed around the thin plate martensite in $\mathrm{Fe}-\mathrm{Ni}-\mathrm{C}$ alloys $^{(5)}$ these dislocations are considered to be associated with the martensite interface when the specimen was in the martensite condition and left behind in the parent phase after reverse transformation.

In specimens which experienced a complete transformation cycle, the region at which the wedge and spear type pairs were joined are recognized from the above dislocations residual in the parent phase on the corresponding transformation twin planes in the martensite variants and on the junction planes. The wedge and spear type pairs can be distinguished by the location of the transformation twin plane normal and the junction plane normal. In the wedge type pair the three normals are nearly $60^{\circ}$ apart; and in the spear type pair the twin plane normals are $120^{\circ}$ apart and the junction plane bisects these planes.

Another structure observed in the specimens after complete reversal is what is supposed to be the junction plane of the pairs of variants in a plate group. The junction plane of a pair of variants which forms a wedge morphology shows two directions of line defects, each of which corresponds to the intersection of the transformation twin planes of each variant and the junction plane. The junction plane of the pair of variants which forms a spear type morphology shows a single direction of line defects which corresponds to the direction of the intersection of the transformation twin planes in both variants and the junction plane. The junction region of the pair of variants which forms a kink type morphology shows a cluster of dislocations along the obtuse angle bisecting plane of the habits of these variants, which indicates that the junction plane of this pair is not a simple plane and that the junction area is associated with relatively heavy deformation.

\section{Ackowledgement}

This work was supported by the U. S. Army Research Office (Durham) and its support is 
gratefully ackowledged.

\section{REFERENCES}

(1) M. Umemoto and C. M. Wayman: Procedings of First JIM Int. Symp. on New Aspects of Martensitic Transformation, Supplement to Trans. JIM, Vol. 17 (1976), p. 193.

(2) S. Kajiwara and W. S. Owen: Met. Trans., 4 (1973), 1988; 5 (1974), 2047.

(3) S. Kajiwara: Procedings of First JIM Int. Symp. on New Aspects of Martensitic Transformation, Supplement to Trans. JIM, Vol. 17 (1976), p. 81.

(4) G. Krauss, Jr.: Acta Met., 11 (1963), 499.

(5) T. Maki and C. M. Wayman: Procedings of First JIM Int. Symp. on New Aspects of Martensitic Transformation, Supplement to Trans. JIM, Vol. 17 (1976), p. 75.

(6) P. B. Hirsh, A. Howie, R. B. Nicholson, D. W.
Pashley and M. J. Whelan: Electron Microscopy of Thin Crystals, London, Butterworths (1965), p. 185.

(7) T. Maki and C. M. Wayman: Proceedings of First JIM Int. Symp. on New Aspects of Martensitic Transformation, Supplement to Trans. JIM, Vol. 17 (1976), p. 69.

(8) M. Watanabe and C. M. Wayman: Met. Trans., 2 (1971), 2221, 2229.

(9) S. Kajiwara and W. S. Owen: Scripta Met., 11 (1977), 137.

(10) M. Umemoto and C. M. Wayman: Acta Met., in press.

(11) S. Chakravorty and C. M. Wayman: Met. Trans., 7A (1976), 555.

(12) T. A. Schroder and C. M. Wayman: Acta Met., in press. 